\title{
Massive stellar systems: observational challenges and perspectives in the E-ELT era
}

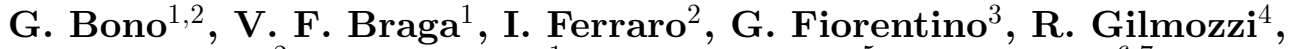 \\ G. Iannicola $^{2}$, D. Magurno ${ }^{1}$, N. Matsunaga ${ }^{5}$, M. Monelli $^{6,7}$ and \\ S. Rastello ${ }^{1}$ \\ ${ }^{1}$ Universitá di Roma "Tor Vergata", Via della Ricerca Scientifica 1, 00133 Roma, Italy \\ email: giuseppe.bono@roma2.infn.it \\ ${ }^{2}$ INAF-Osservatorio Astronomico di Roma, Via Frascati 33, 00040 Monteporzio Catone, Italy \\ ${ }^{3}$ INAF-Osservatorio Astronomico di Bologna, via Ranzani 1, 40127, Bologna, Italy \\ ${ }^{4}$ European Southern Observatory, K. Schwarzschild-Str. 2, 85748 Garching-Munchen, Germany \\ ${ }^{5}$ Dept. of Astronomy, University of Tokyo, 7-3-1 Hongo, Bunkyo-ku, Tokyo 113-0033, Japan \\ ${ }^{6}$ Instituto de Astrofísica de Canarias, La Laguna, Tenerife, Spain \\ ${ }^{7}$ Departamento de Astrofísica, Universidad de La Laguna, Tenerife, Spain
}

\begin{abstract}
We introduce the empirical framework concerning optical and near-infrared (NIR) photometry of crowded stellar fields. In particular, we address the impact that linear detectors and analytical PSF played in improving the accuracy and the precision of multi-band colormagnitude diagrams (CMDs). We focus our attention on recent findings based on deep NIR images collected with Adaptive Optics (AO) systems at the 8-10m class telescopes and discuss pros and cons of the different approaches. We also discuss the estimate of the absolute age of globular clusters using a well defined knee along the lower main sequence. We mention the role which the current AO-assisted instruments will have in addressing longstanding astrophysical problems of the Galactic center. Finally, we outline the role of first generation of E-ELT instruments upon photometry and spectroscopy of crowded stellar fields.
\end{abstract}

Keywords. Galaxy: nucleus, globular clusters: general, techniques: photometric, techniques: spectroscopic, stars: distances, stars: evolution, stars: oscillations

\section{Introduction}

Photometry in crowded stellar fields such as globular clusters (GCs), Galactic Bulge (GB) and the Nuclear Bulge (NB) has been for decades the "battering ram" to constrain their stellar content and to investigate several open astrophysical problems. In this context, accurate absolute stellar ages play a crucial role, since they provide firm constraints on the early formation and evolution of the Galactic spheroid, and on the interaction among the oldest Galactic components (e.g. Vandenberg et al. 1996; Athanassoula 2005). The Galactic bulge has been addressed in many papers (Kunder et al. 2015; Valenti et al. 2015), in the following we focus our attention on GCs and on NB.

Since the pioneering CMDs by Arp et al. (1953) and Sandage (1953) GCs have been the subject of extensive photometric surveys. The advent of CCD cameras during the 80s opened a new path (Schild \& Kent 1981; Kristian \& Blouke 1982): despite their initially small field of view, the new bidimensional sensors, with their high-sensitivity and linear response, allowed for precise photometric measurements of a significantly larger number of individual stars in GCs. However, the new opportunities brought new challenges for the data reduction, and this pushed the development of new algorithms for precise stellar photometry (e.g. DAOPHOT, Stetson (1987); ROMAFOT, Buonanno (1989); DoPhot, Schechter et al. (1993); SExtractor, Bertin \& Arnouts (1996); StarFinder, Diolaiti et al. 
(2000)). Recently, the high spatial resolution provided by space images collected with the Hubble Space Telescope in optical bands allowed us new relevant leaps forward in GC studies and software development (HSTphot, Dolphin (2000); EPSF, Anderson et al. (2008)). They paved the road for collecting homogeneous, high-quality multi-band images for a significant fraction of Galactic (Marín-Franch et al. 2009) and Local Group (LG) (Olszewski et al. 1996; Buonanno et al. 1999; Rich et al. 2005) GCs.

The absolute age of GCs has been studied extensively in the literature (Vandenberg et al. 1990; Chaboyer et al. 1998), since the main sequence turn-off (MSTO) is the fundamental clock provided by stellar evolution. However, so far this method has been hampered by observational uncertainties. The dominant source of error is the GC distance: the current uncertainties are at least $\approx 5 \%$, this means at least $10 \%$ in distance modulus (0.1 mag) and error on absolute age $\geqslant 1$ Gyr (Renzini 1991). The error on the reddening and on the chemical composition also contribute to the age uncertainty.

The above problems become even more severe in the NB, since these regions are extremely crowded and heavily reddened. This is why scientists started studying the stellar content of the NB twenty years ago, with the advent of NIR arrays. Early investigations by Philipp et al. (1999) and Mezger et al. (1999) constrained the contribution of lowand high-mass stars in the dynamical mass (90\% vs 10\%) and in the K-band flux (6\% vs $94 \%)$. The young star clusters: Nuclear Star Cluster, Arches and Quintuplet were investigated using space (NICMOS at HST, Figer et al. (2002)) and ground-based (SINFONI, Martins et al. (2008); Liermann et al. (2010a); NACO at VLT, Espinoza et al. (2009)) NIR and MIR images.

The metallicity distribution of the NB is also in its infancy, and indeed accurate iron abundances for red and blue supergiants (SG) were only recently provided (Martins et al. 2008; Najarro et al. 2009; Davies et al. 2009). The current estimates suggest a roughly solar iron abundance. This finding has been supported by accurate NIR highresolution spectra collected with GIANO at TNG (Origlia et al. 2015). Note that the above metallicities are systematically more metal-poor than Galactic Cepheids in the inner disk (Genovali et al. 2015). This suggests that the NB and the inner disk underwent different chemical-enrichment histories.

The above investigations were complemented by low- and high-resolution NIR spectra collected with ISAAC and with CRIRES at VLT by Ryde \& Schultheis (2015). They investigated nine M-type giants located in the NB, and found that they are metal-rich $([\mathrm{Fe} / \mathrm{H}]=0.11 \pm 0.15)$. Moreover, they noticed that their $\alpha$-element abundances are similar to outer-bulge stars, i.e. relatively $\alpha$-poor. New constraints on the metallicity distribution of the central parsec were provided by Do et al. (2015) using low-resolution spectra for a large sample of RG stars (83) in the NB collected with NIFS at Gemini north. They were the first scientists to find evidence of both metal-poor $([\mathrm{Fe} / \mathrm{H}] \leqslant-1)$ and super metal-rich $([\mathrm{Fe} / \mathrm{H}] \geqslant 0.5)$ stars. These findings open a new path concerning the formation of the NB, i.e. the in situ formation versus the accretion formation.

Note that the NB is, together with the thin disc, the only Galactic region for which we still lack firm identifications of old ( $\mathrm{t} \geqslant 10 \mathrm{Gyr}$ ) stellar populations (Bono et al. 2015).

\section{A new spin on the absolute age of GCs}

The absolute age of GCs are mainly based on the comparison between predicted and observed magnitude of main-sequence turn-off stars (MSTO). Pros and cons of optical and NIR CMDs, together with intrinsic and systematic errors have been discussed in many papers (Girardi et al. 2002; Salaris \& Weiss 2002; Dotter et al. 2009; Vandenberg et al. 2013; Di Cecco et al. 2015; Milone et al. 2015; Monelli et al. 2015). The same 


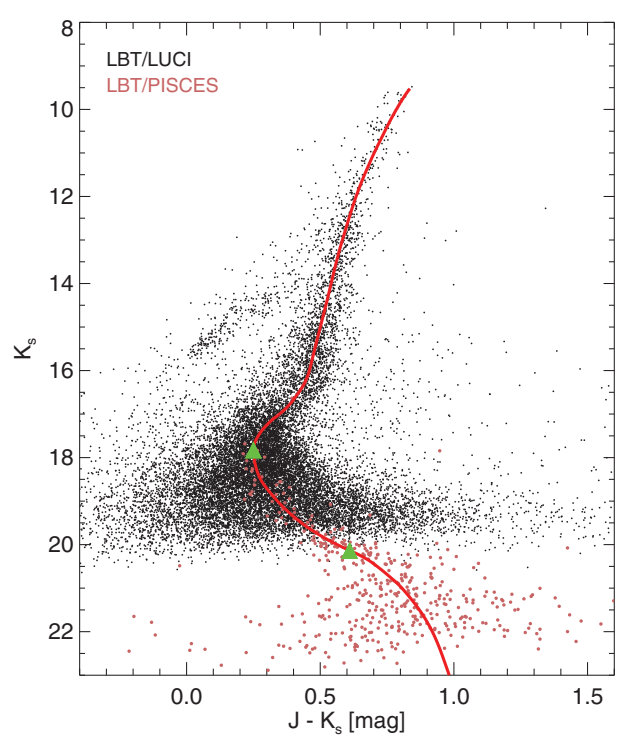

Figure 1. NIR (J,J-K) Color-Magnitude Diagram of the Galactic globular M15. Data were collected seeing limited with LUCI at LBT (black dots) and with the AO system (FLAO) at LBT (red dots) in an off-center field. The red line shows the ridgeline of the cluster, while the bright and faint green triangles mark the main sequence turn-off and the main sequence knee.

applies to the impact that systematics have on relative and absolute ages (Marín-Franch et al. 2009; Bono et al. 2010; Vandenberg et al. 2013).

An independent approach to date GCs, based on a different clock, and in turn on different physics, is the use of the white dwarf cooling sequence. Accurate CMDs provided new firm constraints on the absolute age of GCs (Bedin et al. 2005; Hansen et al. 2013) and old open cluster (Kalirai et al. 2007). The use of the WD luminosity function has solid advantages, when compared with the canonical approach (Salaris et al. 2010). Note that deep and accurate NIR photometry of stellar systems is going to play a crucial role, since the WD cooling sequence shows in the faint end, not only a blue (Hansen et al. 2004), but also a red turn off (Bono et al. 2013). This means new constraints on the absolute age and on the distance of GCs.

A very interesting approach for dating individual and cluster stars is the stellar cosmochronology. Its key advantage is that it relies on stellar spectroscopy only, and is therefore independent of errors affecting distance and reddening corrections. Moreover, a small fraction of extremely metal-poor $([\mathrm{Fe} / \mathrm{H}] \leqslant-2.5)$ stars shows a strong enhancement in neutron-capture elements (r-process) i.e. the channel producing the large majority of heavy elements. Its main problem is the need for accurate measurements of weak, blue lines of ${ }^{232} \mathrm{Th}(4019 \AA$, half-life $14 \mathrm{Gyr})$ and ${ }^{238} \mathrm{U}$ (3859.6 $\AA$, half-life, 4.5 Gyr) together with the stable s-process element Eu (Frebel \& Kratz 2009). This approach has only been applied to a few field stars (Cayrel et al. 2001; Sneden et al. 2003) and to three red giants (RG) in M15 (Sneden et al. 2000). The results are very promising, but still hampered by large errors, indeed $U$ can only be measured using a single optical line.

A new spin on the problem of cluster dating was provided by space and ground-based deep and accurate NIR CMDs. They clearly display a well defined knee along the main sequence (MSK). The bending is due to an atmospheric opacity mechanism: the collisional induced absorption of $\mathrm{H} 2$ molecules (Pulone et al. 1999; Zoccali et al. 2000; Saumon et al. 2012). The difference in magnitude or in color between the MSTO and the MSK 
is a good age indicator, since the MSK is independent of age $\left(\mathrm{M} / \mathrm{M}_{\odot} \sim 0.4-0.5\right)$ and only mildly affected by metallicity (Kalirai 2015).

In this context, NIR images collected with the Multiconjugated Adaptive optics Demonstrator (MAD) available at VLT (Marchetti et al. (2003)) provided the unique opportunity to fully exploit this technique by using ground-based data (Bono et al. 2010). More recently, a modest number of NIR images collected with the AO system available at LBT (First Light Adaptive Optics available at LBT allowed us to constrain with very high accuracy the absolute age of the metal-poor Galactic globular M15. Figure 1 shows the comparison between the data and cluster isochrones, we found an age of $13.3 \pm 1.1 \mathrm{Gyr}$ that agrees within the errors with the age based on the MSTO (12.9 $\pm 2.6 \mathrm{Gyr})$. However, the error is at least a factor of two smaller than similar evaluations in the literature.

Note that the photometry of crowded stellar fields collected with ground-based AO systems is far from being a trivial issue. The key problem is that the shape of the PSF does depend on the distance from the guide stars used to close the AO loop. Note that this problem changes when moving from the J- to $\mathrm{K}$ - band images, and it is also affected by external seeing conditions. The above problems increase when moving from multi-conjugated to single-conjugated AO systems. In order to overcome these problems, different approaches have been suggested, based either on the use of a quadratic change (Bono et al. 2010) of the PSF across the FoV, or individual deconvolutions (Schreiber et al. 2014), or asymmetric PSF (Fiorentino et al. 2014; Monelli et al. 2015).

\section{Nuclear bulge}

The Nuclear Bulge represents the crossroads of several outstanding astrophysical and cosmological open problems. a) There are two competing theories to explain the formation of the GC. In the so-called "in-situ star formation" scenario, the stellar content of the GC builds up with stellar clusters and gas located in its vicinity. In the "accreted" scenario, the stellar content of the NB builds up thanks to the disruption of several GCs due to tidal forces (Capuzzo-Dolcetta \& Miocchi 2008). The former scenario is supported by the presence of the nuclear star cluster and of two very young open clusters: Arches ( $\mathrm{t} \sim 2-2.5$ Myr, Figer et al. (2002); Najarro et al. (2004)) and Quintuplet ( $\mathrm{t} \sim 2.4-5 \mathrm{Myr}$, Figer et al. (1999); Liermann et al. (2010b)).

The period (age) range of a few classical Cepheids recently discovered (Matsunaga et al. 2011) and their kinematic properties (Matsunaga et al. 2015) support the presence of ongoing star formation activity during the last 30 Myra. Dating back to the seminal NIR investigation by Figer et al. (2004) there is also evidence of an intermediate-age stellar population (red clump stars). We still solid evidence of an old stellar population in the NB. Two dozen of type II Cepheids were identified by Matsunaga et al. (2013). These variables are the progeny of hot HB stars, i.e. stars older than 10 Gyr. However, there is mounting empirical evidence that some of them are in binary systems, and their progenitors appear to have ages of the order of a few Gyrs (Soszyński et al. 2011).

Space (HST, SPITZER) and ground based photometric (NAOS/CONICA at VLT) and spectroscopic (KMOS at VLT) data (Schödel et al. 2014; Feldmeier 2015; Ghez 2015) improved the analysis of NIR and MIR CMDs of the NB. However, we still lack firm constraints on the old stellar populations. Numerical simulations indicate that a disk-buckling instability drifted the material (stars, gas) present in the pristine Disk into the Bulge (Athanassoula 1992). This prediction is supported by the lack of old stellar tracers (old open cluster, RRL, HB stars) with Disk kinematics.

The radial distribution of RRLs in the Bulge is well known (Soszyński et al. 2014; Pietrukowicz et al. 2015). However, recent kinematic investigations indicate that RRLs 


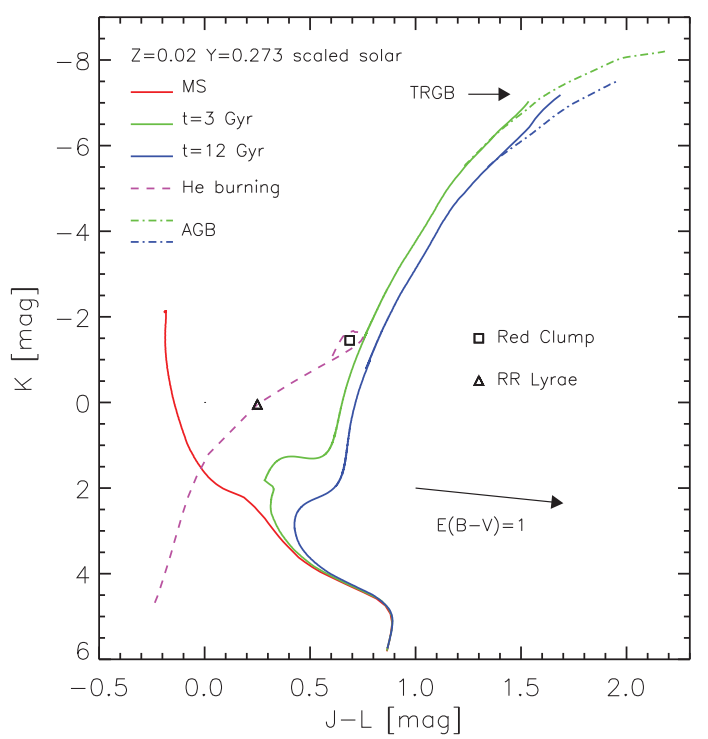

Figure 2. Predicted K,J-L CMD. The isochrones are based on evolutionary tracks (BASTI) computed assuming solar iron abundance (metals, $\mathrm{Z}=0.02$; helium, $\mathrm{Y}=0.273$ ) and a scaled solar chemical mixture (Pietrinferni et al. 2014). The red line displays a young (30 Myr) main sequence (MS), while the green and the blue lines two cluster isochrones for intermediate (3 Gyr) and old (12 Gyr) stellar structures. The purple dashed line shows the helium burning sequence for old and intermediate-age structures, while the dashed-dotted line the Asymptotic Giant Branch (AGB). The black triangle marks the center of the RR Lyrae instability strip, while the black square the Red Clump (RC) stars. The Tip of the Red Giant Branch (TRGB) is marked with a short black arrow. The long black arrow shows the reddening vector for $E(B-V)=1$ mag.

do not rotate like Bulge giants, indeed their mean radial velocities are vanishing independently of the longitude. Moreover, they have larger velocity dispersions than RGs. This means that RRLs do not follow the cylindrical rotation of Bar/Bulge stellar populations. However, photometric and spectroscopic investigations of RRLs in the Bulge stop at 2 degrees from the NB. This means that we lack information concerning old stellar tracers in the transition between the inner Bulge/Bar and the NB.

\section{Paving the road to E-ELT}

While waiting for the next generation of ELTs, there are second and third generation instruments at the $8-10 \mathrm{~m}$ class telescopes, which will pave the way not only for the photometry, but also for the spectroscopy. The MCAO plus four sodium lasers available at Gemini North (GEMS $\dagger$ ) provides superb NIR images of Galactic globulars (Turri et al. 2015). The Fov is $83 \times 83$ arcsec squared with a pixel scale of $0.02 \mathrm{arcsec} /$ pixel and allowed to reach a very good accuracy down to $\mathrm{K} \approx 22 \mathrm{mag}$.

HAWK-I and the new AO Facility at UT4 (GRAAL $\ddagger$ ) are also planned to deliver deep NIR images, but on a larger field of view: 7.5 arcmin square. Note that the pixel scale is 0.11 arcsec and is expected to decrease by almost a factor of two the seeing at optical wavelengths. However, the instrument which is going to play a crucial role in

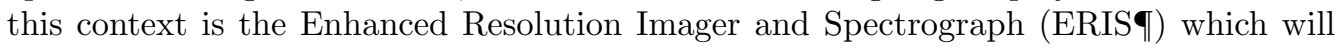

$\dagger$ http://www.gemini.edu/sciops/instruments/gems/ $\ddagger$ http://www.eso.org/sci/facilities/develop/ao/sys/graal.html

I https://www.eso.org/sci/facilities/develop/instruments/eris.html 
become available at UT4 (Amico et al. 2012; Kuntschner, H. et al. 2014). The reason is threefold: a) the field of view (1x1 arcmin square), the pixel scale $(0.027 \mathrm{arcsec} / \mathrm{px})$ and the expected performance of the single-conjugate AO system (Strehl ratio larger than $54 \%$ in K-band, using both a Natural Guide Star [NGS, R 12 mag] and a Na Laser Guide star [LGS]) appear very promising for crowded stellar fields. b) The camera is covering a broad wavelength range $(1-5 \mu \mathrm{m})$, and in turn a very good color sensitivity. c) The unique opportunity to use a NIR integral field unit, SCAO assisted, with a field of view of 8 arcsec square and a medium resolution grating $(\mathrm{R} \sim 8,000)$. This means iron and $\alpha$-element abundances for evolved cool stars in the Galactic centre and Bulge.

To further constrain the impact of this instrument, Fig. 2 shows a K,J-L CMD at solar chemical composition. Theoretical predictions (http://basti.oa-teramo.inaf.it) plotted in this CMD display three interesting features: a) the color sensitivity is $50 \%$ larger than classical K,J-K CMDs, and in turn a better sensitivity in the main sequence turn off (MSTO) region of old ( $\mathrm{K} \sim 3 \mathrm{mag})$ and intermediate ( $\mathrm{K} \sim 2 \mathrm{mag}$ ) stellar ages. b) The occurrence of the MSK at $\mathrm{K} \sim 5$ and J-L $\sim 0.9$ mag. c) The most important old (RR Lyrae, $\mathrm{K} \sim 0 \mathrm{mag}$ ) and intermediate $(\mathrm{RC}, \mathrm{K} \sim-1.5 \mathrm{mag}$ ) age stellar tracers that display half magnitude difference in color.

If we assume that we can routinely reach with ERIS at VLT a limiting magnitude $\mathrm{K} \sim 22-23(10 \sigma)$, then we could also constrain the age distribution not only in inner/outer Bulge and in low reddening regions of the NB, since the expected apparent magnitude of the MSK, assuming a canonical reddening law (Cardelli et al. 1989) is 14.5 (true distance modulus $)+2\left(\mathrm{~A}_{K}\right)+5$ (MSK absolute magnitude) 21.5 mag. Moreover, the opportunity to measure RR Lyrae and RC stars in crowded and reddened central regions of dwarf galaxies in the LG ( $\mu \sim 23 \mathrm{mag})$.

\section{Conclusions and future perspectives}

The GCs and the NB are perfect targets for AO observations with this new generation of instruments, due to their high stellar density: i) they host plenty of bright stars (Rband $<16-17$ mag, blue and red SGs, RGs and Asymptotic Giant Branch stars) that can be used as natural guide stars (NGS) to drive the tip-tilt correction; ii) they represent an ideal test-bed for the current on-sky AO performance and, in turn, to investigate our ability in doing accurate photometry/astrometry using these new images where the Point Spread Function (PSF) changes across the FOV. In particular, in the coming era of ELTs, it becomes crucial to understand whether available data reduction software and techniques will allow us to deal with and to exploit current AO data (Fiorentino et al. 2014).

An important step forward concerning the stellar content of GCs and of the NB is expected in the near future, once the new generation of extremely large ground-based telescopes (GMT, Giant Magellan Telescope [http://www.gmto.org/]; TMT, Thirty Meter Telescope [http://www.tmt.org/]; E-ELT, European Extremely Large Telescope [http://www.eso.org/sci/facilities/eelt/index.html]) equipped with sophisticated AO systems optimized for the NIR bands, will become available (Greggio et al. 2012).

The limiting magnitudes and the volume within which E-ELT will allow us to perform accurate NIR photometry is a quantum jump. Indeed, we can assume limiting magnitudes of $\mathrm{J}, \mathrm{K} \sim 29-30 \mathrm{mag}$. There is an unprecedented opportunity to reach with MICADO (http://www.mpe.mpg.de/ir/micado) RR Lyrae and RC stars in the innermost regions of giant elliptical galaxies in nearby galaxy groups (Sculptor, $\mu \sim 20$; Centaurus A, $\mu \sim 29$; M81 $\mu \sim 28)$. Moreover, the opportunity to reach TRGB $(\mathrm{K} \sim-7 \mathrm{mag})$ and AGB $(\mathrm{K} \sim-8$ mag) stars in Virgo $(\mu \sim 30)$, Eridanus $(\mu \sim 32)$ and Coma $(\mu \sim 35$ mag $)$ cluster. 
Moreover, the E-ELT intergral field spectrograph HARMONI $\dagger$ and the AO assisted IFUs of the E-ELT multi-object spectrograph (MOSAIC $\ddagger$ ) are going to provide a complete spectroscopic analysis $(\mathrm{R} \sim 20,000)$ of the old stellar component $\left(\mathrm{MSTO}, \mathrm{M}_{K} \sim 3\right.$ mag) even in very high-reddened regions of the $\mathrm{NB}\left(\mathrm{A}_{K} \sim 3 \mathrm{mag}, \mathrm{K} \sim 21-22\right)$. The same applies to galaxies in the LG or in its outskirts (Antlia group, $\mu \sim 26$, Karachentsev \& Kashibadze (2005)) using RG and AGB stars. This means a never explored parameter space in the hearts of nearby galaxies. This is a jump in the current understanding of the formation and evolution of galactic bulges, and in particular for their chemical enrichment history. The same applies to the stellar populations in elliptical galaxies for which we only have limited hints from the Galactic bulge and from the dwarf elliptical M32.

\section{Acknowledgements}

GB thanks the Japan Society for the Promotion of Science for a research grant (L15518).

\section{References}

Amico, P. et al. 2012, SPIE, 8446, 20

Anderson, J., Sarajedini, A., Bedin, L. R. et al. 2008, AJ, 135, 2055

Arp, H. C., Baum, W. A., \& Sandage, A. R. 1953, AJ, 58, 4

Athanassoula, E. 1992, MNRAS, 259, 345

Athanassoula, E. 2005, AIPC, 804, 333

Bedin, L. R., Cassisi, S., Castelli, F. et al. 2005, MNRAS, 357, 1038

Bertin, E. \& Arnouts, S. 1996, A\&AS, 117, 393

Bono, G., Genovali, K., Lemasle, B. et al. 2015, ASPC, 491, 148

Bono, G., Salaris, M., \& Gilmozzi, R. 2013, A\&A A, 549, A102

Bono, G., Stetson, P. B., VandenBerg, D. A. et al. 2010, ApJ, 708, L74

Buonanno, R., Corsi, C. E., Castellani, M. et al. 1999, AJ, 118, 1671

Buonanno, R. 1989, ESOC, 31, 183

Capuzzo-Dolcetta, R. \& Miocchi, P. 2008, ApJ, 681, 1136

Cardelli, J. A., Clayton, G. C., \& Mathis, J. S. 1989, ApJ, 345, 245

Cayrel, R., Hill, V., Beers, T. C. et al. 2001, Natur, 409, 691

Chaboyer, B., Demarque, P., Kernan, P. J. et al. 1998, ApJ, 494, 96

Davies, B., Origlia, L., Kudritzki, R.-P. et al. 2009, ApJ, 694, 46

Di Cecco, A., Bono, G., Prada Moroni, P. G. et al. 2015, AJ, 150, 51

Diolaiti, E., Bendinelli, O., Bonaccini, D. et al. 2000, ASPC, 216, 623

Do, T., Kerzendorf, W., Winsor, N. et al. 2015, ApJ, 809, 143

Dolphin, A. E. 2000, PASP, 112, 1383

Dotter, A., Kaluzny, J., \& Thompson, I. B. 2009, IAUS, 258, 171

Espinoza, P., Selman, F. J., \& Melnick, J. 2009, AछA, 501, 563

Feldmeier, A. 2015, IAUGA, 22, 52322

Figer, D. F., Rich, R. M., Kim, S. S. et al. 2004, ApJ, 601, 319

Figer, D. F., Najarro, F., Gilmore, D. et al. 2002, ApJ, 581, 258

Figer, D. F., McLean, I. S., \& Morris, M. 1999, ApJ, 514, 202

Fiorentino, G., Ferraro, I., Iannicola, G. et al. 2014, SPIE, 9148, 91483U

Frebel, A. \& Kratz, K.-L. 2009, IAUS, 258, 449

Genovali, K., Lemasle, B., da Silva, R. et al. 2015, A\&\&A, 580, A17

Ghez, A. 2015, IAUGA, 22, 58584

Girardi, L., Bertelli, G., Bressan, A. et al. 2002, A\&AA, 391, 195

Greggio, L., Falomo, R., Zaggia, S. et al. 2012, PASP, 124, 653

Hansen, B. M. S., Kalirai, J. S., Anderson, J. et al. 2013, Natur, 500, 51

$\dagger$ https://www2.physics.ox.ac.uk/research/visible-and-infrared-instruments/harmoni $\ddagger$ http://www.e-elt.nl/index.php? $\mathrm{v}=$ mosaic 
Hansen, B. M. S., Richer, H. B., Fahlman, G. G. et al. 2004, ApJS, 155, 551

Kalirai, J. 2015, IAUGA, 22, 33338

Kalirai, J. S., Bergeron, P., Hansen, B. M. S. et al. 2007, ApJ, 671, 748

Karachentsev, I. D. \& Kashibadze, O. G. 2005, astro, arXiv:astro-ph/0509207

Kristian, J. \& Blouke, M. 1982, SciAm, 247, 66

Kunder, A., Rich, R. M., Hawkins, K. et al. 2015, ApJ, 808, L12

Kuntshner, H. et al. 2014, SPIE, 9147, 1

Liermann, A., Kraus, M., Schnurr, O. et al. 2010a, MNRAS, 408, L6

Liermann, A., Hamann, W.-R., Oskinova, L. M. et al. 2010b, A\& A, 524, A82

Marchetti, E., Hubin, N. N., Fedrigo, E. et al. 2003, SPIE, 4839, 317

Marín-Franch, A., Aparicio, A., Piotto, G. et al. 2009, ApJ, 694, 1498

Martins, F., Hillier, D. J., Paumard, T. et al. 2008, A\&AA, 478, 219

Matsunaga, N., Fukue, K., Yamamoto, R. et al. 2015, ApJ, 799, 46

Matsunaga, N., Feast, M. W., Kawadu, T. et al. 2013, MNRAS, 429, 385

Matsunaga, N., Kawadu, T., Nishiyama, S. et al. 2011, Natur, 477, 188

Mezger, P. G., Zylka, R., Philipp, S. et al. 1999, A\&A, 348, 457

Milone, A. P., Marino, A. F., Bedin, L. R. et al. 2015, arXiv, arXiv:1510.05086

Monelli, M., Testa, V., Bono, G. et al. 2015, ApJ, 812, 25

Najarro, F., Figer, D. F., Hillier, D. J. et al. 2009, ApJ, 691, 1816

Najarro, F., Figer, D. F., Hillier, D. J. et al. 2004, ApJ, 611, L105

Olszewski, E. W., Suntzeff, N. B., \& Mateo, M. 1996, ARA\&A, 34, 511

Origlia, L., Oliva, E., Sanna, N. et al. 2015, arXiv, arXiv:1510.06870

Philipp, S., Zylka, R., Mezger, P. G. et al. 1999, A\&A, 348, 768

Pietrinferni, A., Molinaro, M., Cassisi, S. et al. 2014, A\&C, 7, 95

Pietrukowicz, P., Kozłowski, S., Skowron, J. et al. 2015, ApJ, 811, 113

Pulone, L., de Marchi, G., \& Paresce, F. 1999, A\&A, 342, 440

Renzini, A. 1991, ASIC, 348, 131

Rich, R. M., Corsi, C. E., Cacciari, C. et al. 2005, AJ, 129, 2670

Ryde, N. \& Schultheis, M. 2015, A\&3A, 573, A14

Salaris, M., Cassisi, S., Pietrinferni, A. et al. 2010, ApJ, 716, 1241

Salaris, M. \& Weiss, A. 2002, A\&BA, 388, 492

Sandage, A. R. 1953, AJ, 58, 61

Saumon, D., Marley, M. S., Abel, M. et al. 2012, ApJ, 750, 74

Schechter, P. L., Mateo, M., \& Saha, A. 1993, PASP, 105, 1342

Schild, R. \& Kent, S. 1981, SPIE, 290, 186

Schödel, R., Feldmeier, A., Kunneriath, D. et al. 2014, A\&A, 566, A47

Schreiber, L., Greggio, L., Falomo, R. et al. 2014, MNRAS, 437, 2966

Sneden, C., Cowan, J. J., Lawler, J. E. et al. 2003, ApJ, 591, 936

Sneden, C., Johnson, J., Kraft, R. P. et al. 2000, ApJ, 536, L85

Soszyński, I., Udalski, A., Szymański, M. K. et al. 2014, AcA, 64, 177

Soszyński, I., Dziembowski, W. A., Udalski, A. et al. 2011, AcA, 61, 1

Stetson, P. B. 1987, PASP, 99, 191

Stolte, A., Hussmann, B., Olczak, C. et al. 2015, yCat, 357, 80004

Stolte, A. \& Brandner, W. 2010, IAUS, 266, 123

Turri, P., McConnachie, A. W., Stetson, P. B. et al. 2015, ApJ, 811, L15

Valenti, E., Zoccali, M., Gonzalez, O. A. et al. 2015, arXiv, arXiv:1510.07425

Vandenberg, D. A., Brogaard, K., Leaman, R. et al. 2013, ApJ, 775, 134

Vandenberg, D. A., Bolte, M., \& Stetson, P. B. 1996, ARA\& $\&$, 34, 461

Vandenberg, D. A., Bolte, M., \& Stetson, P. B. 1990, JRASC, 84, 412

Zoccali, M., Cassisi, S., Frogel, J. A. et al. 2000, ApJ, 530, 418 